\title{
VECTOR-VALUED INEQUALITIES FOR THE COMMUTATORS OF SINGULAR INTEGRALS WITH ROUGH KERNELS
}

\author{
Lin TANg and Huoxiong Wu
}

\begin{abstract}
In this paper, we establish the vector-valued inequalities for the commutators of singular integrals with rough kernels. In particular, our results can essentially improve some well-known results.
\end{abstract}

\section{Introduction}

Let $\mathbb{R}^{n}(n \geq 2)$ be the $n$-dimensional Euclidean space and $S^{n-1}$ be the unit sphere in $\mathbb{R}^{n}$ equipped with normalized Lebesgue measure $d \sigma=d \sigma(x)$. Let $\Omega$ be a homogeneous function of degree zero on $\mathbb{R}^{n}$ and $\Omega(x) \in L^{s}\left(S^{n-1}\right)(s \geq 1)$, and

$$
\int_{S^{n-1}} \Omega(x) d \sigma(x)=0 .
$$

The Calderón-Zygmund singular integral operator $T$ is defined by

$$
T f(x)=p . v \cdot \int_{\mathbb{R}^{n}} \frac{\Omega(y)}{|y|^{n}} f(x-y) d y,
$$

where $f \in C_{0}^{\infty}\left(\mathbb{R}^{n}\right)$.

In their fundamental work on singular integrals, Calderón and Zygmund established the $L^{p}$ boundedness of $T$ for $1<p<\infty$ under the condition that $\Omega \in L \log ^{+} L\left(S^{n-1}\right)$, i.e.,

$$
\int_{S^{n-1}}|\Omega(y)| \log (2+|\Omega(y)|) d \sigma(y)<\infty .
$$

The condition that $\Omega \in L \log ^{+} L\left(S^{n-1}\right)$ turns out to be most desirable size condition for the $L^{p}$ boundedness of $T$. This was made clear by Calderón and Zygmund [5] where it was shown that $T$ may fail to be bounded on $L^{p}$ for any $p$

Received September 20, 2009; Revised April 6, 2010.

2010 Mathematics Subject Classification. 42B20, 42B25, $42 \mathrm{~B} 35$.

Key words and phrases. singular integral, commutator, rough kernel, Fourier transform estimate.

The research was supported by the NNSF(10971002) and NNSF(10571122) of China. 
if the condition $\Omega \in L \log ^{+} L\left(S^{n-1}\right)$ is replaced by any condition $\Omega \in L^{\phi}\left(S^{n-1}\right)$ with a $\phi$ satisfying $\phi(t)=o(t \log t)$ as $t \rightarrow \infty$.

The block space $B_{q}^{0, v}\left(S^{n-1}\right)$ introduced by Lu et al. in [17], have some properties such as: $L^{q}\left(S^{n-1}\right) \subset B_{q}^{0, v}\left(S^{n-1}\right)(-1 \leq v), B_{q}^{0, v_{1}}\left(S^{n-1}\right) \subset B_{q}^{0, v_{2}}\left(S^{n-1}\right)$ for $v_{1}>v_{2}$ and $q>1$, and all inclusions are proper. But the spaces $L(\log L)^{\mu}\left(S^{n-1}\right)$ and $B_{q}^{0, \mu-1}\left(S^{n-1}\right)$ with $0<\mu$ do not contain each other. Jiang and $\mathrm{Lu}[17]$ studies the $L^{p}$ boundedness of singular integral operator $T$ with kernels belonging to certain block spaces. Recently, H. Al-Qassem and Y. Pan [2] established the $L^{p}$ estimates for singular integrals $T$ provided that $\Omega \in B_{q}^{0,0}$ with $q>1$.

On the other hand, the study of vector-valued inequalities for singular integrals with rough kernels $\Omega \in L^{q}\left(S^{n-1}\right)$ with $q>1$ attracted much attention; see [13] and [22]. Naturally, it is an interesting problem whether we can establish the vector-valued inequalities for singular integrals with rough kernels $\Omega$ satisfying weaker conditions. In fact, in this paper, we will consider the vector-valued inequalities for a class of higher order commutators followed by $T_{b, k}$ is defined as follows

$$
T_{b, k} f(x)=p \cdot v \cdot \int_{\mathbb{R}^{n}} \frac{\Omega(x-y)}{|x-y|^{n}}[b(x)-b(y)]^{k} f(y) d y,
$$

where $b \in B M O\left(\mathbb{R}^{n}\right), k \in \mathbb{N}$ and $f \in C_{0}^{\infty}\left(\mathbb{R}^{n}\right)$.

A celebrated result of Coifman and Meyer [8] states that if $\Omega \in C^{1}\left(S^{n-1}\right)$, then for $1<p<\infty, T_{b, 1}$ is bounded on $L^{p}\left(\mathbb{R}^{n}\right)$. When $\Omega \in \cup_{s>1} L^{s}\left(S^{n-1}\right)$, by a well-known result of Duoandikoetxea [9] and the Alvarez-Bagby-Kurtz-Pérez boundedness criterion for the commutators of linear operator ([1], Theorem $2.13)$, we know that the operators $T_{b, k}$ are bounded on $L^{p}$ for $1<p<\infty$. Recently, $\mathrm{Hu}$ [15] proved that if $\Omega \in L(\log L)^{k+1}\left(S^{n-1}\right)$, then the operators $T_{b, k}$ are bounded on $L^{p}$ for $1<p<\infty$. In this paper, we will establish the following results.

Theorem 1.1. Let $\Omega$ be homogeneous of degree zero and satisfy (1.1), $k \in \mathbb{N}$ and $b \in B M O\left(\mathbb{R}^{n}\right), h(r) \in L^{\infty}(0, \infty)$. If $\Omega \in L(\log L)^{k+1}\left(S^{n-1}\right) \bigcup B_{\infty}^{0, k}\left(S^{n-1}\right)$, then the operators $T_{h, b, k}$ defined by

$$
T_{h, b, k} f(x)=p . v \cdot \int_{\mathbb{R}^{n}} h(|x-y|) \frac{\Omega(x-y)}{|x-y|^{n}}[b(x)-b(y)]^{k} f(y) d y,
$$

are bounded on $L^{p}\left(l^{q}\right)$ with bound $C_{p, q}\|b\|_{B M O\left(\mathbb{R}^{n}\right)}^{k}$ provided that $1<p, q<\infty$.

In addition, we also consider the following oscillatory singular integral commutator $\bar{T}_{b, k}$ defined by

$$
\bar{T}_{b, k} f(x)=p . v \cdot \int_{\mathbb{R}^{n}} e^{i p(x, y)} \frac{\Omega(x-y)}{|x-y|^{n}}[b(x)-b(y)]^{k} f(y) d y,
$$

where $p(x, y)$ is a real polynomial on $\mathbb{R}^{n} \times \mathbb{R}^{n}, b \in B M O\left(\mathbb{R}^{n}\right), k \in \mathbb{N}$ and $f \in C_{0}^{\infty}\left(\mathbb{R}^{n}\right)$. 
Theorem 1.2. Let $\Omega$ be homogeneous of degree zero and satisfy (1.1), $k \in \mathbb{N}$ and $b \in B M O\left(\mathbb{R}^{n}\right)$. If $\Omega \in L(\log L)^{k+1}\left(S^{n-1}\right) \cup B_{\infty}^{0, k}\left(S^{n-1}\right)$, then the operators $\bar{T}_{b, k}$ are bounded on $L^{p}\left(l^{q}\right)$ with bound $C_{p, q}\|b\|_{B M O\left(\mathbb{R}^{n}\right)}^{k}$ provided that $1<p, q<\infty$, where $C_{p, q}$ is a constant which depends only on $p, q$ and degree of $p(x, y)$ but not its coefficients.

We remark that Theorems 1.1 and 1.2 are also new even if in the case $k=0$; see [3], [7] and [6]. In particular, it is worth pointing out that the proof of Theorem 1.1 is slightly different from [15], and we obtain Corollary 4.1 by using Theorem 1.2, which improves the main results in [7].

The paper is organized as follows. In Section 2, we will give some preliminary lemmas for Theorem 1.1. Next we will prove Theorem 1.1 in Section 1.3. The proof of Theorem 1.2 will be given in Section 4 .

Throughout this paper, $C$ is a positive constant which is independent of the main parameters and not necessary the same at each occurrence. For a measure set $E$, denote by $\chi_{E}$ the characteristic function of $E$. For $f$ defined on $\mathbb{R}^{n}, \hat{f}$ denotes the Fourier transform of $f$.

We collect the notation to be used throughout this paper:

$$
\begin{gathered}
\|f\|_{L^{p}\left(l^{q}\right)}=\left(\int_{\mathbb{R}^{n}}\left(\sum_{j \in \mathbb{Z}}\left|f_{j}(x)\right|^{q}\right)^{p / q} d x\right)^{1 / p} \text { for } f=\left\{f_{j}\right\}_{j \in \mathbb{Z}} ; \\
\|f\|_{p}=\left(\int_{\mathbb{R}^{n}}|f(x)|^{p} d x\right)^{1 / p} \text { and }\|f\|_{L^{p}(\omega)}=\left(\int_{\mathbb{R}^{n}}|f(x)|^{p} \omega(x) d x\right)^{1 / p} .
\end{gathered}
$$

\section{Some lemmas for Theorem 1.1}

In this section, we fix $a \geq 2$ and $A, B>0$.

Lemma 2.1. Let $\phi \in C_{0}^{\infty}\left(\mathbb{R}^{n}\right)$ be a radial function such that $\operatorname{supp} \phi \subset\{1 / 4 \leq$ $|\xi| \leq 4\}$ and

$$
\sum_{l \in \mathbb{Z}} \phi^{3}\left(a^{-l} \xi\right)=1, \quad|\xi| \neq 0
$$

Define the multiplier operator $S_{l}$ by

$$
\widehat{S_{l} f}(\xi)=\phi\left(a^{-l}\right) \hat{f}(\xi),
$$

and $S_{l}^{2}$ by $S_{l}^{2} f(x)=S_{l}\left(S_{l} f\right)(x)$. For any positive integer $k$ and $b \in B M O\left(\mathbb{R}^{n}\right)$, denote by $S_{l, b, k}$ (respectively $S_{l, b, k}^{2}$ ) the kth order commutator of $S_{l}$ (respectively $\left.S_{l}^{2}\right)$. Then for $1<p, q<\infty$,

(i) $\left\|\left(\sum_{l \in \mathbb{Z}}\left|S_{l, b, k} f\right|^{2}\right)^{1 / 2}\right\|_{L^{p}\left(l^{q}\right)} \leq C(n, k, p, q)\|b\|_{B M O\left(\mathbb{R}^{n}\right)}^{k}\|f\|_{L^{p}\left(l^{q}\right)}$,

(ii) $\left\|\left(\sum_{l \in \mathbb{Z}}\left|S_{l, b, k}^{2} f\right|^{2}\right)^{1 / 2}\right\|_{L^{p}\left(l^{q}\right)} \leq C(n, k, p, q)\|b\|_{B M O\left(\mathbb{R}^{n}\right)}^{k}\|f\|_{L^{p}\left(l^{q}\right)}$, 
(iii) $\left\|\sum_{l \in \mathbb{Z}} S_{l, b, k} f_{l}\right\|_{L^{p}\left(l^{q}\right)} \leq C(n, k, p, q)\|b\|_{B M O\left(\mathbb{R}^{n}\right)}^{k}\left\|\left(\sum_{l \in \mathbb{Z}}\left|f_{l}\right|^{2}\right)^{1 / 2}\right\|_{L^{p}\left(l^{q}\right)}$.

The Lemma 2.1 follows from the weighted Littlewood-Paley theory, extrepolation theorem and the main theorems in [1].

Lemma 2.2. Suppose $\left\{\sigma_{j}\right\}_{j \in \mathbb{Z}}$ is a sequence of Borel measures in $\mathbb{R}^{n}$. Write $\sigma_{j, b, 0} f(x)=\sigma_{j} * f(x)$, and for $k \in \mathbb{N}$,

$$
\sigma_{j, b, k} f(x)=b(x) \sigma_{j, b, k-1} f(x)-\sigma_{j, b, k-1}(b f)(x) .
$$

Suppose for any $1<p, q<\infty$ such that

$$
\left\|\mu_{b, k}^{*} f\right\|_{L^{p}\left(l^{q}\right)}+\left\|\bar{\mu}_{\bar{b}, k}^{*} f\right\|_{L^{p}\left(l^{q}\right)} \leq C B\|b\|_{B M O\left(\mathbb{R}^{n}\right)}^{k}\|f\|_{L^{p}\left(l^{q}\right)},
$$

where $\bar{\mu}_{\bar{b}, k}^{*} f(x)=\sup _{j \in \mathbb{Z}}\left|\bar{\mu}_{j, \bar{b}, k} f(x)\right|, \mu_{j}=\left|\sigma_{j}\right|, \bar{\mu}_{j}(x)=\left|\sigma_{j}\right|(-x)$ and $\bar{b}(x)=$ $b(-x)$. Then the following vector valued inequality

$$
\left\|\left(\sum_{j \in \mathbb{Z}}\left|\sigma_{j, b, k} f_{j}\right|^{2}\right)^{1 / 2}\right\|_{L^{p}\left(l^{q}\right)} \leq C(n, k, p, q) B\|b\|_{B M O\left(\mathbb{R}^{n}\right)}^{k}\left\|\left(\sum_{j \in \mathbb{Z}}\left|f_{j}\right|^{2}\right)^{1 / 2}\right\|_{L^{p}\left(l^{q}\right)}
$$

holds for $1<p, q<\infty$.

Proof. We borrow some ideas from [10]. Since $\sup _{j \in \mathbb{Z}}\left|\sigma_{j, b, k} f_{j l}\right| \leq \mu_{b, k}^{*}\left(\sup _{j \in \mathbb{Z}}\left|f_{j l}\right|\right)$, we then have

$$
\begin{aligned}
& \left\|\left(\sum_{l \in \mathbb{Z}}\left(\sup _{j \in \mathbb{Z}}\left|\sigma_{j, b, k} f_{j l}\right|\right)^{q}\right)^{1 / q}\right\|_{p} \\
\leq & \left\|\left(\sum_{l \in \mathbb{Z}}\left(\mu_{b, k}^{*}\left(\sup _{j \in \mathbb{Z}}\left|f_{j l}\right|\right)\right)^{q}\right)^{1 / q}\right\|_{p} \\
\leq & C(n, k, p, q) B\|b\|_{B M O\left(\mathbb{R}^{n}\right)}^{k}\left\|\left(\sum_{l \in \mathbb{Z}}\left(\sup _{j \in \mathbb{Z}}\left|f_{j l}\right|\right)^{q}\right)^{1 / q}\right\|_{p} .
\end{aligned}
$$

On the other hand, there exists a sequence $\left\{h_{j}\right\} \in L^{p^{\prime}}\left(l^{q^{\prime}}\right)$ such that

$$
\begin{aligned}
& \left\|\left(\sum_{l \in \mathbb{Z}}\left(\sum_{j \in \mathbb{Z}}\left|\sigma_{j, b, k} f_{j l}\right|\right)^{q}\right)^{1 / q}\right\|_{p} \\
\leq & \sup _{\|h\|_{L p^{p^{\prime}\left(l^{\prime}\right)}} \leq 1}\left|\sum_{l \in \mathbb{Z}} \int_{\mathbb{R}^{n}} \sum_{j \in \mathbb{Z}}\right| \sigma_{j, b, k} f_{j l}\left|h_{l}(x) d x\right| \\
\leq & \sup _{\|h\|_{L^{p^{\prime}}\left({ }^{\prime} q^{\prime}\right)} \leq 1} \sum_{l \in \mathbb{Z}} \int_{\mathbb{R}^{n}} \sum_{j \in \mathbb{Z}}\left|f_{j, l}(x)\right| \bar{\mu}_{\bar{b}, k}^{*} h_{l}(x) d x
\end{aligned}
$$




$$
\leq C(n, k, p, q) B\|b\|_{B M O\left(\mathbb{R}^{n}\right)}^{k}\left\|\left(\sum_{l \in \mathbb{Z}}\left(\sum_{j \in \mathbb{Z}}\left|f_{j l}\right|\right)^{q}\right)^{1 / q}\right\|_{p},
$$

where $p^{\prime}=p /(p-1)$ and $q^{\prime}=q /(q-1)$.

Interpolating between above inequalities, we get

$$
\begin{gathered}
\left\|\left(\sum_{l \in \mathbb{Z}}\left(\sum_{j \in \mathbb{Z}}\left|\sigma_{j, b, k} f_{j l}\right|^{2}\right)^{q / 2}\right)^{1 / q}\right\|_{p} \\
\leq C B\|b\|_{B M O\left(\mathbb{R}^{n}\right)}^{k}\left\|\left(\sum_{l \in \mathbb{Z}}\left(\sum_{j \in \mathbb{Z}}\left|f_{j l}\right|^{2}\right)^{q / 2}\right)^{1 / q}\right\|_{p} .
\end{gathered}
$$

Thus, Lemma 2.2 is proved.

Lemma 2.3 (See $[14,15,16])$. Let $m_{\delta} \in C^{1}\left(\mathbb{R}^{n}\right)(0<\delta<\infty)$ be a family of multipliers such that supp $m_{\delta} \subset\{|\xi|<\delta\}$ and for some constants $C$ and $\alpha$,

$$
\left\|m_{\delta}\right\|_{\infty} \leq C A \min \left\{\delta^{-\alpha}, \delta^{\alpha}\right\}, \quad\left\|\nabla m_{\delta}\right\|_{\infty} \leq C A .
$$

Let $T_{\delta}$ be the multiplier operator defined by $\widehat{T_{\delta} f}(\xi)=m_{\delta}(\xi) \hat{f}(\xi)$. For positive integer $k$ and $b \in B M O\left(\mathbb{R}^{n}\right)$, denote by $T_{\delta, b, k}$ the $k$ th order commutator of $T_{\delta}$. Then for any $0<\mu<1$, there exists a positive constant $C=C(n, k, \mu)$ such that

$$
\left\|T_{\delta, b, k} f\right\|_{2} \leq C A \alpha^{-k}\|b\|_{B M O\left(\mathbb{R}^{n}\right)}^{k} \min \left\{\delta^{-\alpha \mu}, \delta^{\alpha \mu}\right\}\|f\|_{2} .
$$

Lemma 2.4. Let $\left\{\sigma_{j}\right\}_{j \in \mathbb{Z}}$ be a sequence of finite Borel measure in $\mathbb{R}^{n}$. Suppose for $j \in \mathbb{Z}$,

$$
\left\|\sigma_{j}\right\| \leq C A, \quad\left\|\nabla \hat{\sigma}_{j}\right\|_{\infty} \leq C A a^{j}
$$

and there exists a positive constant $0<\alpha<1$ such that

$$
\left|\hat{\sigma}_{j}(\xi)\right| \leq C A \min \left\{\left|a^{j} \xi\right|,\left|a^{j} \xi\right|^{-\alpha / \ln a}\right\} .
$$

Suppose $k \in \mathbb{N}$ and nonnegative integer $h \leq k$ such that for any $1<p, q<\infty$

$$
\left\|\mu_{b, h}^{*} f\right\|_{L^{p}\left(l^{q}\right)}+\left\|\bar{\mu}_{\bar{b}, h}^{*} f\right\|_{L^{p}\left(l^{q}\right)} \leq C B\|b\|_{B M O\left(\mathbb{R}^{n}\right)}^{h}\|f\|_{L^{p}\left(l^{q}\right)} .
$$

Then,

$$
T_{b, k} f(x)=\sum_{j \in \mathbb{Z}} \sigma_{j, b, k} f(x)
$$

is a bounded operator in $L^{p}\left(l^{q}\right)$ with bound

$$
C(n, k, p, q)\left(A(\ln a)^{k}\right)^{\theta_{p, q}} B^{1-\theta_{p, q}}\|b\|_{B M O\left(\mathbb{R}^{n}\right)}^{k},
$$

where $0<\theta_{p, q}<1$ depending only on $p$ and $q$. 
Proof. Choose a radial function $\phi \in C_{0}^{\infty}\left(\mathbb{R}^{n}\right)$ such that $0 \leq \phi \leq 1$, supp $\phi \subset$ $\{1 / 4 \leq|\xi| \leq 4\}$ and

$$
\sum_{l \in \mathbb{Z}} \phi^{3}\left(a^{-l}|\xi|\right)=1, \quad|\xi| \neq 0 .
$$

Define the multiplier operator $S_{l}$ by

$$
\widehat{S_{l} f}(\xi)=\phi\left(a^{-l} \xi\right) \hat{f}(\xi) .
$$

Set $m_{j}(\xi)=\hat{\sigma}_{j}(\xi), m_{j}^{l}(\xi)=m_{j}(\xi) \phi\left(a^{j-l} \xi\right)$, and by $(2.1)$, we have

$$
\widehat{T_{j}^{l} f}(\xi)=m_{j}^{l}(\xi) \hat{f}(\xi) .
$$

Obviously, $\operatorname{supp}_{j}^{l}\left(a^{-j} \xi\right) \subset\left\{|\xi| \leq 2 a^{l}\right\}$ and

$$
\left|m_{j}^{l}\left(a^{-j} \xi\right)\right| \leq C A \min \left\{a^{l}, a^{-l \alpha / \ln a}\right\},\left\|\nabla m_{j}^{l}\left(a^{-j} \cdot\right)\right\|_{\infty} \leq C A .
$$

Let $\bar{T}_{j}^{l}$ be the operator defined by

$$
\widehat{\bar{T}_{j}^{l} f}(\xi)=m_{j}^{l}\left(a^{-j} \xi\right) \hat{f}(\xi) .
$$

The inequality (2.2) via Lemma 2.3 says that for $0<\mu<1$,

$$
\left\|\bar{T}_{j, b, k}^{l} f\right\|_{2} \leq C_{\mu} A(\ln a)^{k} a^{-\alpha \mu|l| / \ln a}\|b\|_{B M O\left(\mathbb{R}^{n}\right)}^{k}\|f\|_{2} .
$$

If $b \in B M O\left(\mathbb{R}^{n}\right)$, then $b_{t}(x)=b(t x) \in B M O\left(\mathbb{R}^{n}\right)$ and $\left\|b_{t}\right\|_{B M O\left(\mathbb{R}^{n}\right)}=$ $\|b\|_{B M O\left(\mathbb{R}^{n}\right)}$ for any $t>0$. By dilation invariance, we have

$$
\left\|T_{j, b, k}^{l} f\right\|_{2} \leq C_{\mu} A(\ln a)^{k} a^{-\alpha \mu|l| / \ln a}\|b\|_{B M O\left(\mathbb{R}^{n}\right)}^{k}\|f\|_{2} .
$$

On the other hand, using Plancherel theorem, we have

$$
\left\|T_{j}^{l} f\right\|_{2} \leq C_{\mu} A(\ln a)^{k} a^{-\alpha \mu|l| / \ln a}\|f\|_{2} .
$$

We know from [15] that for any $f, g \in C_{0}^{\infty}\left(\mathbb{R}^{n}\right)$,

$$
\int_{\mathbb{R}^{n}} g(x) T_{b, k} f(x) d x=\int_{\mathbb{R}^{n}} g(x) \sum_{l \in \mathbb{Z}} \sum_{j \in \mathbb{Z}}\left(\left(S_{l-j} T_{j}^{l} S_{l-j}\right)_{b, k} f\right)(x) d x .
$$

With the aid of the formula

$$
(b(x)-b(y))^{k}=\sum_{i=0}^{k} C_{k}^{i}(b(x)-b(z))^{i}(b(z)-b(y))^{k-i}, x, y, z \in \mathbb{R}^{n} .
$$

Let

$$
U_{l} f(x)=\sum_{j \in \mathbb{Z}}\left(\left(S_{l-j} T_{j}^{l} S_{l-j}\right)_{b, k} f\right)(x) .
$$

It is easy to see that

$$
\left(\left(S_{l-j} T_{j}^{l} S_{l-j}\right)_{b, k} f\right)(x)=\sum_{i=0}^{k} C_{k}^{i} S_{l-j, b, k}\left(\left(T_{j}^{l} S_{l-j}\right)_{b, i} f\right)(x) .
$$


Furthermore,

$$
\left(T_{j}^{l} S_{l-j}\right)_{b, i} f(x)=\sum_{h=0}^{i} C_{h}^{i} T_{j, b, h}^{l}\left(S_{l-j, b, i-h} f\right)(x) .
$$

From (2.3), (2.4) and (2.5), we get

$$
\begin{aligned}
& \left\|\left(\sum_{j \in \mathbb{Z}}\left|\left(T_{j}^{l} S_{j-l}\right)_{b, i} f\right|^{2}\right)^{\frac{1}{2}}\right\|_{2}^{2} \\
\leq & C A(\ln a)^{k} a^{-\alpha \mu|l| / \ln a} \sum_{i=0}^{k}\|b\|_{B M O\left(\mathbb{R}^{n}\right)}^{2 h} \sum_{j \in \mathbb{Z}}\left\|S_{l-j, b, i-h} f\right\|_{2}^{2} \\
\leq & C A(\ln a)^{k} a^{-\alpha \mu|l| / \ln a} \sum_{i=0}^{k}\|b\|_{B M O\left(\mathbb{R}^{n}\right)}^{2 i}\|f\|_{2}^{2} .
\end{aligned}
$$

Hence,

$$
\left\|U_{l} f\right\|_{L^{2}\left(l^{2}\right)} \leq C A(\ln a)^{k} a^{-\alpha \mu|l| / \ln a}\|b\|_{B M O\left(\mathbb{R}^{n}\right)}^{k}\|f\|_{L^{2}\left(l^{2}\right)} .
$$

Now let us turn to prove the $L^{p}\left(l^{q}\right)$-boundedness of $U_{l}$. Write

$$
\left(T_{j}^{l} S_{l-j}\right)_{b, i} f(x)=\sum_{h=0}^{i} C_{h}^{i} \sigma_{j, b, h}\left(S_{l-j, b, i-h}^{2} f\right)(x) .
$$

Applying Lemmas 2.1 and 2.2, for any $1<p, q<\infty$, then we have

$$
\begin{aligned}
\left\|U_{l} f\right\|_{L^{p}\left(l^{q}\right)} & \leq C \sum_{i=0}^{k}\|b\|_{B M O\left(\mathbb{R}^{n}\right)}^{k-i}\left\|\left(\sum_{j \in \mathbb{Z}}\left|\left(T_{j}^{l} S_{j-l}\right)_{b, i} f\right|^{2}\right)^{\frac{1}{2}}\right\|_{L^{p}\left(l^{q}\right)} \\
& \leq C \sum_{i=0}^{k}\|b\|_{B M O\left(\mathbb{R}^{n}\right)}^{k-i} \sum_{h=0}^{i}\left\|\left(\sum_{j \in \mathbb{Z}}\left|\sigma_{j, b, h}\left(S_{j-l, b, i-h}^{2} f\right)\right|^{2}\right)^{\frac{1}{2}}\right\|_{L^{p}\left(l^{q}\right)} \\
& \leq C \sum_{i=0}^{k} \sum_{h=0}^{i}\|b\|_{B M O\left(\mathbb{R}^{n}\right)}^{k-i+h}\left\|\left(\sum_{j \in \mathbb{Z}}\left|S_{j-l, b, i-h}^{2} f\right|^{2}\right)^{\frac{1}{2}}\right\|_{L^{p}\left(l^{q}\right)} \\
& \leq C B\|b\|_{B M O\left(\mathbb{R}^{n}\right)}^{k}\|f\|_{L^{p}\left(l^{q}\right)} .
\end{aligned}
$$

In particular, we have

$$
\left\|U_{l} f\right\|_{L^{2}\left(l^{q}\right)} \leq C B\|b\|_{B M O\left(\mathbb{R}^{n}\right)}^{k}\|f\|_{L^{2}\left(l^{q}\right)} .
$$

Interpolating (2.6) and (2.8) for $1<q<\infty$, there exists a constant $0<\theta_{q}<1$ depending only on $q$ such that

$$
\left\|U_{l} f\right\|_{L^{2}\left(l^{q}\right)} \leq C(n, k, p, q)\left(A(\ln a)^{k}\right)^{\theta_{q}} B^{1-\theta_{q}} a^{-\theta_{q} \alpha \mu|l| / \ln a}\|b\|_{B M O\left(\mathbb{R}^{n}\right)}^{k}\|f\|_{L^{2}\left(l^{q}\right)} .
$$

Now, interpolating (2.7) and (2.9), for $1<p<\infty$, there exists a constant $0<\theta_{p, q}<1$ depending only on $p, q$ such that

$$
\left\|U_{l} f\right\|_{L^{p}\left(l^{q}\right)}
$$




$$
\leq C(n, k, p, q)\left(A(\ln a)^{k}\right)^{\theta_{p, q}} B^{1-\theta_{p, q}} a^{-\theta_{p, q} \alpha \mu|l| / \ln a}\|b\|_{B M O\left(\mathbb{R}^{n}\right)}^{k}\|f\|_{L^{p}\left(l^{q}\right)} .
$$

Hence, we have

$$
\left\|T_{b, k} f\right\|_{L^{p}\left(l^{q}\right)} \leq C(n, k, p, q)\left(A(\ln a)^{k}\right)^{\theta_{p, q}} B^{1-\theta_{p, q}}\|b\|_{B M O\left(\mathbb{R}^{n}\right)}^{k}\|f\|_{L^{p}\left(l^{q}\right)} .
$$

This completes the proof of Lemma 2.4.

\section{The proof of Theorem 1.1}

To prove Theorem 1, we consider two cases on $\Omega$.

Case 1. When $\Omega \in L(\log L)^{k+1}\left(S^{n-1}\right)$. As in [3], let $\Omega$ be an integrable function on $S^{n-1}$ satisfying (1.1). Let $E_{m}=\left\{y \in S^{n-1}: 2^{m}<|\Omega(y)| \leq 2^{m+1}\right\}$ for $m \in \mathbb{N}$ and

$$
A(\Omega)=\left\{m \in \mathbb{N}: \sigma\left(E_{m}\right)>2^{-4 m}\right\} .
$$

For each $m \in A(\Omega)$, let

$$
\Omega_{m}=\|\Omega\|_{L^{1}\left(E_{m}\right)}^{-1}\left[\Omega \chi_{E_{m}}-\int_{E_{m}} \Omega d \sigma\right] .
$$

Then the following hold for all $m$ in $A(\Omega)$ :

$$
\begin{gathered}
\int_{S^{n-1}} \Omega_{m} d \sigma=0 ; \\
\left\|\Omega_{m}\right\|_{L^{1}\left(S^{n-1}\right)} \leq 2 ; \\
\left\|\Omega_{m}\right\|_{L^{2}\left(S^{n-1}\right)} \leq 2^{2 m+2} .
\end{gathered}
$$

In addition, we have the following decomposition

$$
\Omega=\Omega_{0}+\sum_{m \in A(\Omega)}\|\Omega\|_{L^{1}\left(E_{m}\right)} \Omega_{m}
$$

where $\Omega_{0} \in L^{2}\left(S^{n-1}\right)$ and satisfies

$$
\int_{S^{n-1}} \Omega_{0} d \sigma=0
$$

For each $m \in A(\Omega) \bigcup\{0\}$, we define

$$
T_{j, b, k}^{(m)} f(x)=p . v \int_{\mathbb{R}^{n}} h(|x-y|) \frac{\Omega_{m}(x-y)}{|x-y|^{n}}[b(x)-b(y)]^{k} f(y) d y .
$$

From (3.1), we know that

$$
T_{h, b, k} f(x)=T_{b, k}^{(0)} f(x)+\sum_{m \in A(\Omega)}\|\Omega\|_{L^{1}\left(E_{m}\right)} T_{b, k}^{(m)} f(x) .
$$

For $m \in A(\Omega)$, let $\left\{\sigma_{j}^{(m)}\right\}_{j \in \mathbb{Z}}$ be the sequence of measure defined by

$$
\int_{\mathbb{R}^{n}} f d \sigma_{j}^{(m)}=\int_{2^{m(j-1)} \leq|y|<2^{m j}} h(|y|) f(y) \frac{\Omega_{m}(y)}{|y|^{n}} d y,
$$


and

$$
\int_{\mathbb{R}^{n}} f d \sigma_{j}^{(0)}=\int_{2^{j-1} \leq|y|<2^{j}} h(|y|) f(y) \frac{\Omega_{0}(y)}{|y|^{n}} d y .
$$

Let $\left|\sigma_{j}^{(m)}\right|$ be defined in the same way as $\sigma_{j}^{(m)}$, but with $\Omega_{m}$ replaced by $\left|\Omega_{m}\right|$. Al-Salman and Pan [3] proved the following result.

Lemma 3.1. Let $h \in L^{\infty}(0, \infty)$. Then there exist constant $\alpha>0$ and $C$ such that

$$
\left|\left(\sigma_{j}^{(m)}\right) \hat{(\xi)}\right|+\mid\left(\left|\sigma_{j}^{(m)}\right| \hat{)}(\xi) \mid \leq C m\left(2^{m j}|\xi|\right)^{-\alpha / m}, \quad m \in A(\Omega)\right.
$$

and

$$
\left|\left(\sigma_{j}^{(0)}\right) \hat{(\xi)}\right|+\left|\left(\left|\sigma_{j}^{(0)}\right|\right) \hat{(\xi)}\right| \leq C\left(2^{j}|\xi|\right)^{-\alpha} .
$$

On the other hand, it is easy to see that

$$
\begin{gathered}
\left.\mid\left(\sigma_{j}^{(m)}\right) \hat{(} \xi\right)\left|\leq C m 2^{m j}\right| \xi \mid, \quad m \in A(\Omega), \\
\left|\nabla\left(\sigma_{j}^{(m)}\right) \hat{(\xi} \xi\right|+\left|\nabla\left(\left|\sigma_{j}^{(m)}\right| \hat{)} \xi\right)\right| \leq C m 2^{m j}, \quad m \in A(\Omega), \\
\left.\mid\left(\sigma_{j}^{(0)}\right) \hat{(} \xi\right)\left|\leq C 2^{j}\right| \xi \mid, \\
\left|\nabla\left(\sigma_{j}^{(0)}\right) \hat{(\xi} \xi\right|+\mid \nabla\left(\left|\sigma_{j}^{(0)}\right| \hat{)}(\xi) \mid \leq C 2^{j},\right.
\end{gathered}
$$

and

$$
\left\|\sigma_{j}^{(m)}\right\| \leq C m, m \in A(\Omega), \quad\left\|\sigma_{j}^{(0)}\right\| \leq C .
$$

We will also need the following key lemma.

Lemma 3.2. Let $k \in \mathbb{N}$ and $b \in B M O\left(\mathbb{R}^{n}\right), \bar{\Omega}$ be homogeneous of degree zero and belong to $L^{\infty}\left(S^{n-1}\right)$. Define the operator $M_{\bar{\Omega}, b, k}$ by

$$
M_{\bar{\Omega}, b, k} f(x)=\sup _{r>0} r^{-n} \int_{|x-y|<r}|b(x)-b(y)|^{k}|\bar{\Omega}(x-y) f(y)| d y .
$$

If $\bar{\Omega} \in L^{\infty}\left(S^{n-1}\right)$, then the maximal operator $M_{\bar{\Omega}, b, k}$ is bounded on $L^{p}\left(l^{q}\right)$ with bound $C_{p, q} \lambda_{\bar{\Omega}, k}\|b\|_{B M O\left(\mathbb{R}^{n}\right)}$ for $1<p, q<\infty$, where $\lambda_{\bar{\Omega}, k}$ defined by

$$
\lambda_{\bar{\Omega}, k}=\inf \left\{\lambda>0: \frac{\|\bar{\Omega}\|_{1}}{\lambda} \log ^{k}\left(2+\frac{\|\bar{\Omega}\|_{\infty}}{\lambda}\right) \leq 1\right\},
$$

where $\|\bar{\Omega}\|_{1}=\|\bar{\Omega}\|_{L^{1}\left(S^{n-1}\right)}$ and $\|\bar{\Omega}\|_{\infty}=\|\bar{\Omega}\|_{L^{\infty}\left(S^{n-1}\right)}$.

Proof. As in [15], without loss of generality, we may assume that $\lambda_{\bar{\Omega}, k}=1$. It is obvious that

$$
\|\bar{\Omega}\|_{1} \log ^{m}\left(2+\|\bar{\Omega}\|_{\infty}\right) \leq 1 .
$$

Let $\widetilde{\phi}_{k}(t)=t \log ^{k}(2+t)$ for $t>0$. Then

$$
\left\|\widetilde{\phi}_{k}(|\bar{\Omega}|)\right\|_{1} \leq 1 \text {. }
$$


Thus,

$$
\begin{aligned}
M_{\bar{\Omega}, b, k} f(x) \leq & \sup _{r>0} r^{-n} \int_{|x-y|<r} \widetilde{\phi}_{k}(|\bar{\Omega}(x-y)|)|f(y)| d y \\
& +C \sup _{r>0} r^{-n} \int_{|x-y|<r} \widetilde{\phi}_{k}(|\bar{\Omega}(x-y)|) e^{|b(x)-b(y)|}|f(y)| d y \\
\leq & \sup _{r>0} r^{-n} \int_{|x-y|<r} \widetilde{\phi}_{k}(|\bar{\Omega}(x-y)|)|f(y)| d y \\
& +C \sup _{r>0} r^{-n} \int_{|x-y|<r} e^{b(x)-b(y)}|f(y)| d y \\
& +C \sup _{r>0} r^{-n} \int_{|x-y|<r} e^{b(y)-b(x)}|f(y)| d y \\
:= & I(f)(x)+I I(f)(x)+I I I(f)(x) .
\end{aligned}
$$

For the first term, by the method of rotation of Calderón and Zygmund [5] and the vector-valued inequality of Hardy-Littlewood maximal operator of Fefferman and Stein [11], we obtain

$$
\|I(f)\|_{L^{p}\left(l^{q}\right)} \leq C\left\|\widetilde{\phi}_{k}(|\bar{\Omega}|)\right\|_{1}\|f\|_{L^{p}\left(l^{q}\right)} .
$$

It remains two terms. By the John-Nireberg inequality, we know that there exist positive constants $A$ and $B$ such that for any cube $Q$,

$$
\frac{1}{|Q|} \int_{Q} \exp \left(\frac{\left|b(x)-b_{Q}\right|}{A\|b\|_{B M O\left(\mathbb{R}^{n}\right)}}\right) d x \leq B
$$

where $b_{Q}$ is the mean value of $b$ on the cube $Q$. Let $C_{1}=\left(A \max \left\{p, p^{\prime}\right\}\right)^{-1}$. Straightforward computation shows that for real-valued $b \in B M O\left(\mathbb{R}^{n}\right)$ with $\|b\|_{B M O\left(\mathbb{R}^{n}\right)}=C_{1}$,

$$
\frac{1}{|Q|} \int_{Q} e^{p\left(b(x)-b_{Q}\right)} d x \leq B, \quad \frac{1}{|Q|} \int_{Q} e^{-p^{\prime}\left(b(x)-b_{Q}\right)} d x \leq B,
$$

and so $e^{p b(x)} \in A_{p}$ (the Muckenhoupt weight class) with the $A_{p}$ constant no more that $C_{2}=B^{p}$. By the weighted vector-valued inequality for the HardyLittlewood maximal operator (see [4]), we have

$$
\|I I(f)\|_{L^{p}\left(l^{q}\right)}+\|I I I(f)\|_{L^{p}\left(l^{q}\right)} \leq C\|f\|_{L^{p}\left(l^{q}\right)} .
$$

Then

$$
\left\|M_{\bar{\Omega}, b, k} f\right\|_{L^{p}\left(l^{q}\right)} \leq C\|f\|_{L^{p}\left(l^{q}\right)} .
$$

Lemma 3.2 is proved.

Let us prove Theorem 1.1 continuously. Let $\mu_{t}^{(m)}=\left|\sigma_{t}^{(m)}\right|, \bar{\mu}_{t}^{(m)}=\left|\sigma_{t}^{(m)}\right|(-x)$ and $\bar{b}(x)=b(-x)$, define the maximal operator by

$$
\left(\sigma^{(m)} f\right)_{b, k}^{*}(x)=\sup _{j \in \mathbb{Z}} \mu_{j, b, k}^{(m)}|f|(x) \quad \text { and } \quad\left(\bar{\sigma}^{(m)} f\right)_{\bar{b}, k}^{*}(x)=\sup _{j \in \mathbb{Z}} \bar{\mu}_{j, \bar{b}, k}^{(m)}|f|(x) .
$$


By Lemma 3.2, for nonnegative integer $h \leq k$, we have

$$
\begin{aligned}
\left\|\left(\sigma^{(m)} f\right)_{b, h}^{*}\right\|_{L^{p}\left(l^{q}\right)}+\left\|\left(\bar{\sigma}^{(m)} f\right)_{\bar{b}, h}^{*}\right\|_{L^{p}\left(l^{q}\right)} & \leq m \lambda_{\Omega_{m}, h}\|b\|_{B M O\left(\mathbb{R}^{n}\right)}^{h}\|f\|_{L^{p}\left(l^{q}\right)} \\
& \leq C m^{k+1}\|b\|_{B M O\left(\mathbb{R}^{n}\right)}^{h}\|f\|_{L^{p}\left(l^{q}\right)} .
\end{aligned}
$$

Let $A_{m}=m, a_{m}=2^{m}$ and $B_{m}=m^{k+1}$, from (3.3)-(3.7) and applying Lemmas 2.4 and 3.1 , we get for $1<p, q<\infty$,

$$
\left\|T_{b, k}^{(0)} f\right\|_{L^{p}\left(l^{q}\right)} \leq C\|b\|_{B M O\left(\mathbb{R}^{n}\right)}^{k}\|f\|_{L^{p}\left(l^{q}\right)},
$$

and

$$
\left\|T_{b, k}^{(m)} f\right\|_{L^{p}\left(l^{q}\right)} \leq C m^{k+1}\|b\|_{B M O\left(\mathbb{R}^{n}\right)}^{k}\|f\|_{L^{p}\left(l^{q}\right)}, \quad m \in A(\Omega) .
$$

Finally, by (3.2), (3.8) and (3.9), we obtain for $1<p, q<\infty$

$$
\begin{aligned}
\left\|T_{h, b, k} f\right\|_{L^{p}\left(l^{q}\right)} & \leq C\left(\left\|T_{b, k}^{(0)} f\right\|_{L^{p}\left(l^{q}\right)}+\sum_{m \in A(\Omega)}\|\Omega\|_{L^{1}\left(E_{m}\right)}\left\|T_{b, k}^{(m)} f\right\|_{L^{p}\left(l^{q}\right)}\right) \\
& \leq C\|b\|_{B M O\left(\mathbb{R}^{n}\right)}^{k}\left(\|f\|_{L^{p}\left(l^{q}\right)}+\sum_{m \in A(\Omega)} m^{k+1}\|\Omega\|_{L^{1}\left(E_{m}\right)}\|f\|_{L^{p}\left(l^{q}\right)}\right) \\
& \leq C\|b\|_{B M O\left(\mathbb{R}^{n}\right)}^{k}\left(1+\|\Omega\|_{L(\log L)^{k+1}\left(S^{n-1}\right)}\right)\|f\|_{L^{p}\left(l^{q}\right)} .
\end{aligned}
$$

Thus, Theorem 1.1 is established if $\Omega \in L(\log L)^{k+1}\left(S^{n-1}\right)$.

Case 2. When $\Omega \in B_{\infty}^{0, k}\left(S^{n-1}\right)$. Let us begin with the definition of $q$-block functions on $S^{n-1}$. We say that a measurable function $b$ on $S^{n-1}$ is a $q$-block if it satisfies the following:

(i) $\operatorname{supp}(b) \subset I$, where and $I$ is an interval on $S^{n-1}$; i.e.,

$$
I=\left\{x^{\prime} \in S^{n-1}:\left|x^{\prime}-x_{0}^{\prime}\right|<\rho\right\} \text { for some } x_{0}^{\prime} \in S^{n-1}, \rho>0 .
$$

(ii) $\|b\|_{q} \leq|I|^{-1 / q^{\prime}}$, where $1 / q+1 / q^{\prime}=1$.

The function space $B_{q}^{0, v}\left(S^{n-1}\right)(v \geq-1), 1<q \leq \infty$, consists of all functions $\Omega \in L^{1}\left(S^{n-1}\right)$ of the form $\Omega=\sum_{\mu=1}^{\infty} c_{\mu} b_{\mu}$ where $c_{\mu} \in \mathbb{C}, b_{\mu}$ is a $q$-block supported in an interval $I_{\mu}$ on $S^{n-1}$ for each $\mu$, and

$$
M_{q}^{0, v}\left(\left\{c_{\mu}\right\}\right)=\sum_{\mu=1}^{\infty}\left|c_{\mu}\right|\left(1+\left(\log \left|I_{\mu}\right|^{-1}\right)^{v+1}\right)<\infty .
$$

For a $q$-block function $b$ on $S^{n-1}$ supported in an interval $I$ with $q>1$ and $\|b\|_{q} \leq|I|^{-1 / q^{\prime}}$ we define the function $\bar{b}$ on $S^{n-1}$ by

$$
\bar{b}(x)=b(x)-\int_{S^{n-1}} b(u) d \sigma(u) .
$$

Then one can easily see that $\bar{b}$ enjoys the following properties:

$$
\begin{gathered}
\int_{S^{n-1}} \bar{b}(u) d \sigma(u)=0 \\
\|\bar{b}\|_{q} \leq 2|I|^{-1 / q^{\prime}}
\end{gathered}
$$




$$
\|\bar{b}\|_{1} \leq 2 .
$$

Let $\bar{b}$ be a blocklike function defined as in (3.11). Define the measures $\left\{\sigma_{\bar{b}: j}\right\}_{j \in \mathbb{Z}}$ by

$$
\int_{\mathbb{R}^{n}} f d \sigma_{\bar{b}, j}=\int_{2^{j-1} \leq|u|<2^{j}} f(u) \frac{\bar{b}(u)}{|u|^{n}} h(|u|) d u .
$$

These measures will be useful only in the case $|I| \geq e^{-2}$ where $I$ is the support of $b$. On the other hand, for the case $|I|<e^{-2}$ we need to define the following measures.

$$
\int_{\mathbb{R}^{n}} f d \lambda_{\bar{b}, j}=\int_{w^{j-1} \leq|u|<w^{j}} f(u) \frac{\bar{b}(u)}{|u|^{n}} h(|u|) d u,
$$

where $w=2^{\left[\log \left(|I|^{-1}\right)\right]}, \quad|I|<e^{-2}$ and $[\cdot]$ denotes the greatest integer function.

Lemma 3.3 (See [3]). Let $\bar{b}$ be a function defined as in (3.11). If $|I|<e^{-2}$, then there are $0<\alpha<1$ and $C>0$ such that

$$
\left|\hat{\lambda}_{b, j}(\xi)\right| \leq C \log \left(|I|^{-1}\right)\left(w^{j}|\xi|\right)^{-\frac{\alpha}{\log \left(|I|^{-1}\right)}} .
$$

By assumption, $\Omega$ can be written as $\Omega=\sum_{\mu=1}^{\infty} c_{\mu} b_{\mu}$ where $c_{\mu} \in \mathbb{C}, b_{\mu}$ is a $\infty$-block supported in an interval $I_{\mu}$ on $S^{n-1}$ and $M_{q}^{0, k}\left(\left\{c_{\mu}\right\}\right)$ satisfies (3.10). For each $\mu=1,2, \ldots$, let $\bar{b}_{\mu}$ be the blocklike function corresponding to $b_{\mu}$. By the vanishing condition on $\Omega$ we have

$$
\Omega=\sum_{\mu=1}^{\infty} c_{\mu} \bar{b}_{\mu}
$$

and hence

$$
\left\|T_{h, b, k} f\right\|_{L^{p}\left(l^{q}\right)} \leq \sum_{\mu=1}^{\infty}\left|c_{\mu}\right|\left\|T_{h, \mu, b, k} f\right\|_{L^{p}\left(l^{q}\right)}
$$

where

$$
T_{h, \mu, b, k} f(x)=p \cdot v \cdot \int_{\mathbb{R}^{n}} h(|x-y|) \frac{\bar{b}_{\mu}(x-y)}{|x-y|^{n}}[b(x)-b(y)]^{k} f(y) d y .
$$

Let $\left\{\sigma_{j}\right\}_{j \in \mathbb{Z}}$ be the sequence of measure defined by

$$
\int_{\mathbb{R}^{n}} f d \sigma_{j}=\int_{2^{j-1} \leq|y|<2^{j}} h(|y|) f(y) \frac{\bar{b}_{\mu}(y)}{|y|^{n}} d y .
$$

Then by (3.11)-(3.14) we have

$$
\begin{gathered}
\left\|\sigma_{j}\right\|+\left\|\left|\sigma_{j}\right|\right\| \leq C \\
\left|\nabla\left(\sigma_{j}\right) \hat{(\xi)}\right|+\left|\nabla\left(\left|\sigma_{j}\right| \hat{)} \xi\right)\right| \leq C 2^{j} \\
\left|\left(\sigma_{j}\right) \hat{(\xi)}\right|+\mid\left(\left|\sigma_{j}\right| \hat{)}(\xi) \mid \leq C\left(2^{j}|\xi|\right)^{-\alpha}\right. \\
\left|\left(\sigma_{j}\right) \hat{(\xi)}\right| \leq C 2^{j}|\xi|
\end{gathered}
$$


for $\left|I_{\mu}\right| \geq e^{-2}$. Also, by (3.11)-(3.14) and Lemma 3.3, we have

$$
\begin{gathered}
\left\|\lambda_{j}^{\mu}\right\|+\left\|\left|\lambda_{j}^{\mu}\right|\right\| \leq C A_{\mu} ; \\
\left.\left|\nabla\left(\lambda_{j}^{\mu}\right) \hat{(\xi)}\right|+\mid \nabla\left(\left|\lambda_{j}^{\mu}\right|\right) \hat{(\xi}\right) \mid \leq C A_{\mu} a_{\mu}^{j} ; \\
\left|\left(\lambda_{j}^{\mu} \hat{)}(\xi)|+|\left(\left|\lambda_{j}^{\mu}\right|\right) \hat{(\xi}\right)\right| \leq C A_{\mu}\left(\omega_{\mu}^{j}|\xi|\right)^{-\alpha / \log \omega_{\mu}} ; \\
\left|\left(\lambda_{j}^{\mu}\right) \hat{(\xi)}\right| \leq C A_{\mu} \omega_{\mu}^{j}|\xi|,
\end{gathered}
$$

where $A_{\mu}=\left[\log \left(\left|I_{\mu}\right|^{-1}\right)\right]^{k+1}$ and $\left|I_{\mu}\right|<e^{-2}$.

Let $\sigma_{j}^{\mu}=\left|\lambda_{j}^{\mu}\right|, \bar{\sigma}_{j}^{\mu}(x)=\left|\lambda_{j}^{\mu}\right|(-x)$ and $\bar{b}(x)=b(-x)$, define the maximal operator by

$$
\sigma_{\mu, b, k}^{*} f(x)=\sup _{j \in \mathbb{Z}} \sigma_{j, b, k}^{\mu}|f|(x) \quad \text { and } \quad \bar{\sigma}_{\mu, \bar{b}, k}^{*} f(x)=\sup _{j \in \mathbb{Z}} \bar{\sigma}_{j, \bar{b}, k}^{\mu}|f|(x) .
$$

If nonnegative integer $h \leq k$, by Lemma 3.2, we get for any $1<p, q<\infty$,

$$
\begin{aligned}
& \left\|\sigma_{\mu, b, h}^{*} f\right\|_{L^{p}\left(l^{q}\right)}+\left\|\bar{\sigma}_{\mu, \bar{b}, h}^{*} f\right\|_{L^{p}\left(l^{q}\right)} \\
\leq & C\left[\log \left(1 /\left|I_{\mu}\right|+2\right)\right] \lambda_{\Omega_{I_{\mu}, h}}\|b\|_{B M O\left(\mathbb{R}^{n}\right)}^{h}\|f\|_{L^{p}\left(l^{q}\right)} \\
\leq & C\left[\log \left(1 /\left|I_{\mu}\right|+2\right)\right]\left(\left[\log \left(1 /\left|I_{\mu}\right|+2\right)\right]^{h}\|\Omega\|_{1}+1\right)\|b\|_{B M O\left(\mathbb{R}^{n}\right)}^{h}\|f\|_{L^{p}\left(l^{q}\right)} \\
\leq & C\left[\log \left(1 /\left|I_{\mu}\right|+2\right)\right]^{k+1}\|b\|_{B M O\left(\mathbb{R}^{n}\right)}^{h}\|f\|_{L^{p}\left(l^{q}\right)} .
\end{aligned}
$$

From these, by Lemmas 2.2, 2.3 and 2.4 , we obtain

$$
\left\|T_{h, \mu, b, k} f\right\|_{L^{p}\left(l^{q}\right)} \leq C\left[\log \left(1 /\left|I_{\mu}\right|+2\right)\right]^{k+1}\|f\|_{L^{p}\left(l^{q}\right)} .
$$

Hence, Theorem 1.1 is proved.

Next we give a result in the case $k=0$ of Theorem 1.1.

Theorem 3.1. Let $\Omega$ be homogeneous of degree zero and satisfy (1.1), $s \in \mathbb{R}$ and $1<p, q<\infty, h(r) \in L^{\infty}(0, \infty)$. If $\Omega \in B_{r}^{0,0}\left(S^{n-1}\right)$ with $r>1$, then the operators $T_{h}$ defined by

$$
T_{h} f(x)=p . v \cdot \int_{\mathbb{R}^{n}} h(|x-y|) \frac{\Omega(x-y)}{|x-y|^{n}} f(y) d y,
$$

are bounded on $L^{p}\left(l^{q}\right)$ with bound $C_{p, q}$.

Adapting the proof of Theorem 1.1, Theorem 3.1 is directly deduced by the following fact that (see [6]): Let $\Omega \in L^{1}\left(S^{n-1}\right)$ and $1<p, q<\infty$. Then (3.15)

$$
\left\|\left(\sum_{j \in \mathbb{Z}}\left|M_{\Omega}\left(f_{j}\right)\right|^{q}\right)^{1 / q}\right\|_{L^{p}\left(\mathbb{R}^{n}\right)} \leq C_{p, q}\|\Omega\|_{L^{1}\left(S^{n-1}\right)}\left\|\left(\sum_{j \in \mathbb{Z}}\left|f_{j}\right|^{q}\right)^{1 / q}\right\|_{L^{p}\left(\mathbb{R}^{n}\right)},
$$

where $C_{p, q}$ is independent of $f_{j}$ for all $j \in \mathbb{Z}$ and $M_{\Omega}$ defined by

$$
M_{\Omega} f(x)=\sup _{r>0} \frac{1}{r^{n}} \int_{|x-y|<r}|\Omega(x-y) f(y)| d y .
$$




\section{The proof of Theorem 1.2}

To prove Theorem 1.2, we will need the following lemmas.

Lemma 4.1. Let $k \in \mathbb{N}$ and $\Omega \in L(\log L)^{k}\left(S^{n-1}\right) \bigcup B_{\infty}^{0, k-1}\left(S^{n-1}\right)$ be homogeneous of degree zero. Suppose that $b \in \mathrm{BMO}\left(\mathbb{R}^{n}\right)$ and $1<p, q<\infty$. Then there exists a positive constant $C$ such that

$$
\left\|M_{\Omega, b, k} f\right\|_{L^{p}\left(l^{q}\right)} \leq C\|b\|_{B M O\left(\mathbb{R}^{n}\right)}^{k}\|f\|_{L^{p}\left(l^{q}\right)},
$$

where $M_{\Omega, b, k} f(x)=\sup _{r>0} r^{-n} \int_{|x-y|<r}\left|\Omega(x-y)[b(x)-b(y)]^{k} f(y)\right| d y$.

Proof. If $\Omega \in L(\log L)^{k}\left(S^{n-1}\right)$, write

$$
\Omega_{l}(x)=\Omega(x) \chi_{E_{l}(x)}
$$

with

$$
\begin{gathered}
E_{0}=\left\{x \in S^{n-1}:|\Omega(x)|<1\right\}, \\
E_{l}=\left\{x \in S^{n-1}: 2^{l-1} \leq|\Omega(x)|<2^{l}\right\}, \quad l \in \mathbb{N} .
\end{gathered}
$$

By Lemma 3.2, we have

$$
\left\|M_{\Omega_{l}, b, k}\right\|_{L^{p}\left(l^{q}\right)} \leq C \lambda_{\Omega_{l, k}}\|b\|_{B M O\left(\mathbb{R}^{n}\right)}^{k}\|f\|_{L^{p}\left(l^{q}\right)} .
$$

Hence,

$$
\left\|M_{\Omega, b, k}\right\|_{L^{p}\left(l^{q}\right)} \leq C \sum_{l \geq 0} \lambda_{\Omega_{l, k}}\|b\|_{\mathrm{BMO}\left(\mathbb{R}^{n}\right)}^{k}\|f\|_{L^{p}\left(l^{q}\right)} \leq C\|b\|_{\mathrm{BMO}\left(\mathbb{R}^{n}\right)}^{k}\|f\|_{L^{p}\left(l^{q}\right)},
$$

where we use the following fact that (see [15])

$$
\sum_{l \geq 0} \lambda_{\Omega_{l, k}} \leq C\left(1+\|\Omega\|_{L(\log L)^{k}\left(S^{n-1}\right)}\right) .
$$

If $\Omega \in B_{\infty}^{0, k-1}\left(S^{n-1}\right)$, without loss of generality, we assume $\Omega \subset I$ is a $\infty$-block. By Lemma 3.2, we have

$$
\left\|M_{\Omega, b, k}\right\|_{L^{p}\left(l^{q}\right)} \leq C[\log (1 /|I|+2)]^{k}\|f\|_{L^{p}\left(l^{q}\right)} .
$$

This completes the proof.

Lemma 4.2. Let $k \in \mathbb{N}$ and $\Omega \in L(\log L)^{k}\left(S^{n-1}\right) \bigcup B_{\infty}^{0, k-1}\left(S^{n-1}\right)$ be homogeneous of degree zero. Suppose that $b \in \operatorname{BMO}\left(\mathbb{R}^{n}\right), k \in \mathbb{N}$ and $1<p, q<\infty$. If the operator

$$
T_{b, k} f(x)=p \cdot v \cdot \int_{\mathbb{R}^{n}} k(x, y)[b(x)-b(y)]^{k} f(y) d y
$$

is bounded on $L^{p}\left(l^{q}\right)$ with bound $C B\|b\|_{\mathrm{BMO}\left(\mathbb{R}^{n}\right)}^{k}$, and $k(x, y)$ satisfies

$$
|k(x, y)| \leq \frac{|\Omega(x-y)|}{|x-y|^{n}},
$$

then the truncated operator

$$
T_{b, k}^{1} f(x)=\int_{|x-y|<1} k(x, y)[b(x)-b(y)]^{k} f(y) d y
$$


is bounded on $L^{p}\left(l^{q}\right)$ with bound $C(B+1)\|b\|_{\mathrm{BMO}\left(\mathbb{R}^{n}\right)}^{k}$.

Proof. Without loss of generality, we may assume that $\|b\|_{\mathrm{BMO}\left(\mathbb{R}^{n}\right)}=1$. For each fixed $h \in \mathbb{R}^{n}$, we split $f=f_{1}+f_{2}+f_{3}$, where

$$
f_{1}(y)=f(y) \chi_{|y-h|<1 / 2}(y), \quad f_{2}(y)=f(y) \chi_{1 / 4<|y-h|<5 / 4}(y) .
$$

It is easy to verify that if $|x-h|<1 / 4$, then

$$
T_{b, k} f_{1}(x)=T_{b, k}^{1} f_{1}(x)=\int_{|x-y|<1} k(x, y)[b(x)-b(y)]^{k} f_{1}(y) d y .
$$

Thus

$$
\int_{|x-h|<1 / 4}\left(\sum_{j=1}^{\infty}\left|T_{b, k}^{1} f_{1 j}(x)\right|^{q}\right)^{p / q} d x \leq B^{p}\|b\|_{\mathrm{BMO}\left(\mathbb{R}^{n}\right)}^{p k}\left\|f_{1}\right\|_{L^{p}\left(l^{q}\right)}^{p} .
$$

If $|x-h|<1 / 4$ and $1 / 2 \leq|y-h|<5 / 4$, then $1 / 4<|x-y|<3 / 2$. So we see that for $|x-h|<1 / 4$,

$\left|T_{b, k}^{1} f_{2}(x)\right| \leq \int_{1 / 4<|x-y|<3 / 2} \frac{|\Omega(x-y)|}{|x-y|^{n}}\left|[b(x)-b(y)]^{k} f_{2}(y)\right| d y \leq C M_{3 / 2, b, k} f_{2}(x)$.

Lemma 4.1 now tells us that

$$
\int_{|x-h|<1 / 4}\left(\sum_{j=1}^{\infty}\left|T_{b, k}^{1} f_{2 j}(x)\right|^{q}\right)^{p / q} d x \leq C\|b\|_{\mathrm{BMO}\left(\mathbb{R}^{n}\right)}^{p k}\left\|f_{2}\right\|_{L^{p}\left(l^{q}\right)}^{p} .
$$

Obviously, we have $T_{b, k}^{1} f_{3}(x)=0$ for $|x-h|<1 / 4$. Combining the above inequalities leads to

$$
\begin{aligned}
& \int_{|x-h|<1 / 4}\left(\sum_{j=1}^{\infty}\left|T_{b, k}^{1} f_{1 j}(x)\right|^{q}\right)^{p / q} d x \\
\leq & C\left(1+B^{p}\right)\|b\|_{\mathrm{BMO}\left(\mathbb{R}^{n}\right)}^{p k} \int_{|x-h|<2}\left(\sum_{j=1}^{\infty}\left|f_{j}(x)\right|^{q}\right)^{p / q} d x .
\end{aligned}
$$

Integrating the last inequality with respect to $h$ gives

$$
\left\|T_{b, k}^{1} f\right\|_{L^{p}\left(l^{q}\right)} \leq C(B+1)\|b\|_{\mathrm{BMO}\left(\mathbb{R}^{n}\right)}^{k}\|f\|_{L^{p}\left(l^{q}\right)} .
$$

This completes the proof of Lemma 4.2.

Proof of Theorem 1.2. Without loss of generality, we may assume that $\|b\|_{\text {BMO }}$ $=1$. We shall argue induction on the degree of the polynomial in $x$ and $y$. If the polynomial $p(x, y)=P_{1}(x)+P_{2}(y)$, Theorem 1.2 is obvious by Theorem 1.1. 
Let $u$ and $v$ be two positive integers and suppose the polynomial has degree $u$ in $x$ and $v$ in $y$. Write

$$
p(x, y)=\sum_{|\alpha| \leq u,|\beta| \leq v} a_{\alpha, \beta} x^{\alpha} y^{\beta} .
$$

By dilation invariance, we may assume that $\sum_{|\alpha|=u,|\beta|=v}\left|a_{\alpha \beta}\right|=1$. Decompose

$$
\begin{aligned}
\bar{T}_{b, k} f(x)= & \int_{|x-y|<1} e^{i p(x, y)} \frac{\Omega(x-y)}{|x-y|^{n}}[b(x)-b(y)]^{k} f(y) d y \\
& +\sum_{d=1}^{\infty} \int_{2^{d-1} \leq|x-y|<2^{d}} e^{i p(x, y)} \frac{\Omega(x-y)}{|x-y|^{n}}[b(x)-b(y)]^{k} f(y) d y \\
= & T_{0} f(x)+\sum_{d=1}^{\infty} T_{d} f(x) .
\end{aligned}
$$

We first consider $T_{0}$. We claim that

$$
\left\|T_{0} f\right\|_{L^{p}\left(l^{q}\right)} \leq C\|f\|_{L^{p}\left(l^{q}\right)} .
$$

We assume that (4.1) holds for all polynomials which are sums of monomials of degree less than $u$ in $x$ times monomials of any degree in $y$, together with monomials which are of degree $u$ in $x$ times monomials which are of degree less than $v$ in $y$. Rewrite

$$
p(x, y)=\sum_{|\alpha|=u,|\beta|=v} a_{\alpha \beta}\left(x^{\alpha} y^{\beta}-y^{\alpha+\beta}\right)+p_{0}(x, y),
$$

where $p_{0}(x, y)$ satisfies the inductive assumption. It follows that

$$
\begin{aligned}
T_{0} f(x)= & \int_{|x-y|<1} e^{i p_{0}(x, y)} \frac{\Omega(x-y)}{|x-y|^{n}}[b(x)-b(y)]^{k} f(y) d y \\
& +\int_{|x-y|<1}\left(e^{i p(x, y)}-e^{i p_{0}(x, y)}\right) \frac{\Omega(x-y)}{|x-y|^{n}}[b(x)-b(y)]^{k} f(y) d y \\
= & T_{0}^{1} f(x)+T_{0}^{2} f(x) .
\end{aligned}
$$

Using inductive assumption based on Theorem 1.1 and Lemma 4.2, we have

$$
\left\|T_{0}^{1} f\right\|_{L^{p}\left(l^{q}\right)} \leq C\|f\|_{L^{p}\left(l^{q}\right)} .
$$

Set $\bar{f}(y)=f(y) \chi_{\{|y| \leq 2\}}$. It is easy to see $T_{0}^{2} f(x)=T_{0}^{2} \bar{f}(x)$ for $|x| \leq 1$. Thus, when $|x| \leq 1$,

$$
\begin{aligned}
T_{0}^{2} f(x) & \leq C \int_{|x-y|<1} \frac{|\Omega(x-y)|}{|x-y|^{n-1}}\left|[b(x)-b(y)]^{k} \bar{f}(y)\right| d y \\
& \leq C \sum_{d=-\infty}^{0} 2^{d} 2^{-d n} \int_{2^{d-1} \leq|x-y|<2^{d}}|\Omega(x-y)||b(x)-b(y)|^{k}|\bar{f}(y)| d y
\end{aligned}
$$




$$
\leq C \sum_{d=-\infty}^{0} 2^{d} M_{2^{d}, b, k} \bar{f}(x) .
$$

By Lemma 4.1, we get

$$
\begin{aligned}
\left(\int_{|x| \leq 1}\left(\sum_{j=1}^{\infty}\left|T_{0}^{2} f_{j}(x)\right|^{q}\right)^{p / q} d x\right)^{1 / p} & \leq C \sum_{d=-\infty}^{0} 2^{d}\left\|M_{2^{d}, b, k} \bar{f}\right\|_{L^{p}\left(l^{q}\right)} \\
& \leq C \sum_{d=-\infty}^{0} 2^{d}\|\bar{f}\|_{L^{p}\left(l^{q}\right)} \\
& \leq C\left(\int_{|y| \leq 2}\left(\sum_{j=1}^{\infty}\left|f_{j}(y)\right|^{q}\right)^{p / q} d y\right)^{1 / p},
\end{aligned}
$$

from which the same argument as that in p. 189 of [20] shows that the inequality

$$
\left(\int_{|x-h| \leq 1}\left(\sum_{j=1}^{\infty}\left|T_{0}^{2} f_{j}(x)\right|^{q}\right)^{p / q} d x\right)^{1 / p} \leq C\left(\int_{|y-h| \leq 2}\left(\sum_{j=1}^{\infty}\left|f_{j}(y)\right|^{q}\right)^{p / q} d y\right)^{1 / p}
$$

holds for all $h \in \mathbb{R}^{n}$ and $C>0$ is independent of $h$. Integrating the last inequality with respect to $h$ and using Hölder's inequality, we finally obtain

$$
\left\|T_{0}^{2} f\right\|_{L^{p}\left(l^{q}\right)} \leq C\|f\|_{L^{p}\left(l^{q}\right)} .
$$

Now we return to consider $T_{d}, d \geq 1$. we consider two cases on $\Omega$.

Case 1. When $\Omega \in L(\log L)^{k+1}\left(S^{n-1}\right)$. Split

$$
T_{d} f(x)=\sum_{l=0}^{\infty} T_{d, l}^{b, k} f(x)
$$

where

with

$$
\begin{gathered}
T_{d, l}^{b, k} f(x)=\int_{2^{d-1} \leq|x-y|<2^{d}} e^{i p(x, y)} \frac{\Omega(x-y)}{|x-y|^{n}}[b(x)-b(y)]^{k} f(y) d y, \\
\Omega_{l}(x)=\Omega(x) \chi_{E_{l}(x)}
\end{gathered}
$$

$$
\begin{gathered}
E_{0}=\left\{x \in S^{n-1}:|\Omega(x)|<1\right\}, \\
E_{l}=\left\{x \in S^{n-1}: 2^{l-1} \leq|\Omega(x)|<2^{l}\right\}, \quad l \in \mathbb{N} .
\end{gathered}
$$

Set $\|\Omega\|_{r}=\|\Omega\|_{L^{r}\left(S^{n-1}\right)}$ for $1 \leq r \leq \infty$ in the rest of this section. If we can prove that for some $\delta>0$,

$$
\left\|T_{d, l}^{b, k} f\right\|_{L^{p}\left(l^{q}\right)} \leq C 2^{-\delta d}\left\|\Omega_{l}\right\|_{\infty}\|f\|_{L^{p}\left(l^{q}\right)},
$$

and

$$
\left\|T_{d, l}^{b, k} f\right\|_{L^{p}\left(l^{q}\right)} \leq C \lambda_{\Omega_{l}, k}\|f\|_{L^{p}\left(l^{q}\right)},
$$


then, for a suitably chosen integer $M>\delta^{-1}$, we have

$$
\begin{aligned}
& \left\|\sum_{l=0}^{\infty} T_{d, l}^{b, k} f\right\|_{L^{p}\left(l^{q}\right)} \\
\leq & \sum_{d=1}^{\infty} \sum_{l=0}^{\infty}\left\|T_{d, l}^{b, k} f\right\|_{L^{p}\left(l^{q}\right)} \\
= & \sum_{d=1}^{\infty}\left\|T_{d, 0}^{b, k} f\right\|_{L^{p}\left(l^{q}\right)}+\sum_{l=1}^{\infty} \sum_{1 \leq d<M l}\left\|T_{d, l}^{b, k} f\right\|_{L^{p}\left(l^{q}\right)}+\sum_{l=1}^{\infty} \sum_{d \geq M l}\left\|T_{d, l}^{b, k} f\right\|_{L^{p}\left(l^{q}\right)} \\
\leq & C\left\|\Omega_{0}\right\|_{\infty}\|f\|_{L^{p}\left(l^{q}\right)}+C \sum_{l=1}^{\infty} l \lambda_{\Omega_{l}, k}\|f\|_{L^{p}\left(l^{q}\right)}+\sum_{l=1}^{\infty} \sum_{d \geq M l} 2^{-\delta l} 2^{l}\|f\|_{L^{p}\left(l^{q}\right)} \\
\leq & C\left(1+\int_{S^{n-1}}|\Omega(x)| \log ^{k+1}(2+|\Omega(x)|) d \sigma(x)\right)\|f\|_{L^{p}\left(l^{q}\right)} .
\end{aligned}
$$

Inequality (4.3) can be seen from Lemma 3.2. To prove (4.2), define

$$
\bar{T}_{d, l}^{b, k} f(x)=\int_{1 \leq|x-y|<2} e^{i p\left(2^{d-1} x, 2^{d-1} y\right)} \frac{\Omega_{l}(x-y)}{|x-y|^{n}}[b(x)-b(y)]^{k} f(y) d y,
$$

and

$$
\bar{T}_{d, l} f(x)=\int_{1 \leq|x-y|<2} e^{i p\left(2^{d-1} x, 2^{d-1} y\right)} \frac{\Omega_{l}(x-y)}{|x-y|^{n}} f(y) d y .
$$

By dilation invariance, it is enough to prove that

$$
\left\|\bar{T}_{d, l}^{b, k} f\right\|_{L^{p}\left(l^{q}\right)} \leq C 2^{-\delta d}\left\|\Omega_{l}\right\|_{\infty}\|f\|_{L^{p}\left(l^{q}\right)} .
$$

By an almost orthogonality argument, we may assume that $f$ has support in a cube $Q$ with side length 1 . Let $\phi \in C_{0}^{\infty}\left(\mathbb{R}^{n}\right), 0 \leq \phi \leq 1$, and let $\phi$ be identically one on $10 \sqrt{n} Q$ and vanish outside of $50 \sqrt{n} Q$, define $\bar{b}(y)=\left(b(y)-m_{\bar{Q}}(f)\right) \phi(y)$, where $m_{\bar{Q}}(f)=|\bar{Q}|^{-1} \int_{\bar{Q}} f$. When $y \in Q$ and $x$ in the support of $T_{d, l} f$, we have

$$
(b(x)-b(y))^{k}=\sum_{m=0}^{k} C_{k}^{m} \bar{b}^{m}(x) \bar{b}_{k-m}(y) .
$$

Write

$$
\bar{T}_{d, l}^{b, k} f(x)=\sum_{m=0}^{k} C_{k}^{m} \bar{b}^{m}(x) \bar{T}_{d, l}\left(\bar{b}^{k-m} f\right)(x) .
$$

For each fixed integer $m, 0 \leq m \leq k$, observe that $\bar{T}_{d, l}\left(\bar{b}^{k-m} f\right) \subset 20 n Q$. We first show that for $1<q<2$ and $q^{\prime}$ the dual index of $q$, then

$$
\left\|\bar{T}_{d, l}\right\|_{q^{\prime}} \leq C 2^{-\delta d}\left\|\Omega_{l}\right\|_{\infty}\|f\|_{q} .
$$

In fact, from the proof in [19], it is easy to see that

$$
\left\|\bar{T}_{d, l} f\right\|_{\infty} \leq C\left\|\Omega_{l}\right\|_{\infty}\|f\|_{1}
$$


and

$$
\left\|\bar{T}_{d, l} f\right\|_{2} \leq C 2^{-\epsilon d}\left\|\Omega_{l}\right\|_{\infty}\|f\|_{2}
$$

where $\epsilon$ is some positive number. By interpolation, we obtain (4.5).

For $1<q<2,1 / q^{\prime}+1 / p_{0}=1 / p$, and $1 / p_{1}+1 / p=1 / q$, by Hölder inequality and (4.5), we get

$$
\begin{aligned}
\left\|\bar{b}^{m} \bar{T}_{d, l}\left(\bar{b}^{k-m} f\right)\right\|_{p} & \leq C\left\|\bar{b}^{m}\right\|\left\|_{p_{0}}\right\| \bar{T}_{d, l}\left(\bar{b}^{k-m} f\right) \|_{q^{\prime}} \\
& \leq C 2^{-\delta d}\left\|\Omega_{d}\right\|_{\infty}\left\|\bar{b}^{k-m} f\right\|_{q} \\
& \leq C 2^{-\delta d}\left\|\Omega_{d}\right\|_{\infty}\left\|\bar{b}^{k-m}\right\|_{p_{1}}\|f\|_{p} \\
& \leq C 2^{-\delta d}\left\|\Omega_{d}\right\|_{\infty}\|f\|_{p}
\end{aligned}
$$

Summing over $m$, we obtain

$$
\left\|\bar{T}_{d, l}^{b, k} f\right\|_{L^{p}\left(l^{p}\right)} \leq C 2^{-\delta d}\left\|\Omega_{l}\right\|_{\infty}\|f\|_{L^{p}\left(l^{p}\right)} .
$$

Obviously, for $\omega \in A_{p}$, we have

$$
\left\|\bar{T}_{d, l}^{b, k} f\right\|_{L^{p}(\omega)} \leq C\left\|\Omega_{l}\right\|_{\infty}\|f\|_{L^{p}(\omega)} .
$$

From this, by extrepolation theorem of $A_{p}$ (see [12]), we get for any $1<q<\infty$,

$$
\left\|\bar{T}_{d, l}^{b, k} f\right\|_{L^{p}\left(l^{q}\right)} \leq C\left\|\Omega_{l}\right\|_{\infty}\|f\|_{L^{p}\left(l^{q}\right)} .
$$

Interpolating (4.6) and (4.7), we obtain (4.4).

Case 2. If $\Omega \in B_{\infty}^{0, k}\left(S^{n-1}\right)$, we consider the terms $T_{d}, d \geq 0$. Without loss of generality, we assume $\Omega \subset I$ is a $\infty$-block. Therefore, we only need to prove that

Split

$$
\left\|T_{d} f\right\|_{L^{p}\left(l^{q}\right)} \leq C[\log (1 /|I|+2)]^{k+1}\|f\|_{L^{p}\left(l^{q}\right)} .
$$

$$
T_{d} f(x)=\sum_{d=0}^{\infty} T_{d}^{b, k} f(x)=\sum_{d=0}^{N} T_{d}^{b, k} f(x)+\sum_{d=N+1}^{\infty} T_{d}^{b, k} f(x)
$$

where

$$
T_{d}^{b, k} f(x)=\int_{2^{d-1} \leq|x-y|<2^{d}} e^{i p(x, y)} \frac{\Omega(x-y)}{|x-y|^{n}}[b(x)-b(y)]^{k} f(y) d y,
$$

and $N$ will be decided later.

From the proof of Lemma 4.1, we know that

$$
\left\|\sum_{d=0}^{N} T_{d}^{b, k} f\right\|_{L^{p}\left(l^{q}\right)} \leq C N[\log (1 /|I|+2)]^{k}\|f\|_{L^{p}\left(l^{q}\right)} .
$$

Now to consider the case $d>N$, define

and

$$
\bar{T}_{d}^{b, k} f(x)=\int_{1 \leq|x-y|<2} e^{i p\left(2^{d-1} x, 2^{d-1} y\right)} \frac{\Omega(x-y)}{|x-y|^{n}}[b(x)-b(y)]^{k} f(y) d y,
$$

$$
\bar{T}_{d} f(x)=\int_{1 \leq|x-y|<2} e^{i p\left(2^{d-1} x, 2^{d-1} y\right)} \frac{\Omega(x-y)}{|x-y|^{n}} f(y) d y .
$$


We claim that

$$
\left\|\bar{T}_{d}^{b, k} f\right\|_{L^{p}\left(l^{q}\right)} \leq C 2^{-\delta d}\|\Omega\|_{q}\|f\|_{L^{p}\left(l^{q}\right)} .
$$

By an almost orthogonality argument, we may assume that $f$ has support in a cube $Q$ with side length 1 . Let $\phi \in C_{0}^{\infty}\left(\mathbb{R}^{n}\right), 0 \leq \phi \leq 1$, and let $\phi$ be identically one on $10 \sqrt{n} Q$ and vanish outside of $50 \sqrt{n} Q$, define $\bar{b}(y)=\left(b(y)-m_{\bar{Q}}(f)\right) \phi(y)$, where $m_{\bar{Q}}(f)=|\bar{Q}|^{-1} \int_{\bar{Q}} f$. When $y \in Q$ and $x$ in the support of $T_{d} f$, we have

$$
(b(x)-b(y))^{k}=\sum_{m=0}^{k} C_{k}^{m} \bar{b}_{m}(x) \bar{b}_{k-m}(y) .
$$

Write

$$
\bar{T}_{d}^{b, k} f(x)=\sum_{m=0}^{k} C_{k}^{m} \bar{b}_{m}(x) \bar{T}_{d}\left(\bar{b}^{k-m} f\right)(x) .
$$

For each fixed integer $m, 0 \leq m \leq k$, observe that $\bar{T}_{d, l}\left(\bar{b}^{k-m} f\right) \subset 20 n Q$. We first notice that for $0<\gamma<n$

$$
\left|\bar{T}_{d} f(x)\right| \leq \int_{1 \leq|x-y|<2} \frac{|\Omega(x-y)|}{|x-y|^{n-\gamma}}|b(x)-b(y)|^{k} f(y) d y .
$$

For any $\sigma>0$ such that $1 /(p+\sigma)=1 / p-\gamma / n$ and $r>n /(n-\gamma)$, we then have

$$
\left\|\bar{T}_{d} f\right\|_{p+\sigma} \leq C\|\Omega\|_{r}\|f\|_{p} .
$$

On the other hand, from the proof in [19], it is easy to see that for $1<p<\infty$

$$
\left\|\bar{T}_{d} f\right\|_{p} \leq C 2^{-\epsilon d}\|\Omega\|_{r}\|f\|_{p},
$$

where $\epsilon$ is some positive number.

By interpolation, by (4.9) and (4.10), we have

$$
\mid \bar{T}_{d} f\left\|_{p} \leq C 2^{-\theta d}\right\| \Omega\left\|_{r}\right\| f \|_{p-\sigma},
$$

where $\theta$ is some positive number.

Let $1 / p_{1}+1 / p_{0}=1 / p$, and $1 /\left(p_{1}-\sigma\right)=1 / p_{2}+1 / p$, by Hölder inequality and (4.11), we get

Summing over $m$, we obtain

$$
\begin{aligned}
\left\|\bar{b}^{m} \bar{T}_{d}\left(\bar{b}^{k-m} f\right)\right\|_{p} & \leq C\left\|\bar{b}^{m}\right\|\left\|_{p_{0}}\right\| \bar{T}_{d}\left(\bar{b}^{k-m} f\right) \|_{p_{1}} \\
& \leq C 2^{-\theta d}\|\Omega\|_{r}\left\|\bar{b}^{k-m} f\right\|_{p_{1}-\sigma} \\
& \leq C 2^{-\theta d}\|\Omega\|_{r}\left\|\bar{b}^{k-m}\right\|_{p_{2}}\|f\|_{p} \\
& \leq C 2^{-\theta d}\|\Omega\|_{r}\|f\|_{p}
\end{aligned}
$$

$$
\left\|\bar{T}_{d}^{b, k} f\right\|_{L^{p}\left(l^{p}\right)} \leq C 2^{-\theta d}\|\Omega\|_{r}\|f\|_{L^{p}\left(l^{p}\right)} .
$$

From [1] and by Theorem 3 of [22], we get for any $1<q<\infty$,

$$
\left\|\bar{T}_{d}^{b, k} f\right\|_{L^{p}\left(l^{q}\right)} \leq C\|\Omega\|_{r}\|f\|_{L^{p}\left(l^{q}\right)} .
$$

Interpolating (4.12) and (4.13), we obtain (4.8). 
Hence, taking $N=2[\log (1 /|I|+2)] / \theta$, we get

$$
\begin{aligned}
& \left\|\sum_{d=0}^{\infty} T_{d}^{b, k} f\right\|_{L^{p}\left(l^{q}\right)} \\
\leq & C N[\log (1 /|I|+2)]^{k}\|f\|_{L^{p}\left(l^{q}\right)}+\sum_{d=N+1}^{\infty} 2^{-\theta d}|I|^{-1 / r^{\prime}}\|f\|_{L^{p}\left(l^{q}\right)} \\
\leq & C[\log (1 /|I|+2)]^{k+1}\|f\|_{L^{p}\left(l^{q}\right)} .
\end{aligned}
$$

Thus, Theorem 1.2 is proved.

Next we give some results in the case $k=0$ of Theorem 1.2.

Theorem 4.1. Let $\Omega$ be homogeneous of degree zero and satisfy (1.1), $s \in \mathbb{R}$ and $1<p, q<\infty$. If $\Omega \in B_{r}^{0,0}\left(S^{n-1}\right)$ with $r>1$ and the operators $T_{P}$ defined by

$$
T_{P} f(x)=p \cdot v \cdot \int_{\mathbb{R}^{n}} e^{i P(x, y)} \frac{\Omega(x-y)}{|x-y|^{n}} f(y) d y,
$$

then

$$
\left\|T_{P} f\right\|_{L^{p}\left(l^{q}\right)} \leq C\|f\|_{L^{p}\left(l^{q}\right)},
$$

where $C_{p, q}$ is a constant which depends only on $p, q$ and degree of the real polynomial $P(x, y)$ but not its coefficients.

Adapting the proof of Theorem 1.2, Theorem 4.1 will be directly deduced by (3.15) and Theorem 3.1.

We remark that in the scalar-valued case, Theorem 4.1 has been proved by A. Al-Salman, H. Al-Qassem, L. C. Chen and Y. Pan [3] if $p(x, y)=p(x-y)$ and $\mathrm{Wu}[18]$ for general real polynomial $P(x, y)$. It should be pointed out that our proof is different from [3] and [18].

As a consequence of Theorems 1.2 and 4.1, we have the following result.

Corollary 4.1. Let $\Omega$ be homogeneous of degree zero and satisfy (1.1), $s \in \mathbb{R}$ and $1<p, q<\infty$. If $\Omega \in L \log ^{+} L\left(S^{n-1}\right) \bigcup B_{r}^{0, k}\left(S^{n-1}\right)$ with $r>1$, then the operators $T_{P}$ defined by

$$
T_{P} f(x)=p . v \cdot \int_{\mathbb{R}^{n}} e^{i P(x-y)} \frac{\Omega(x-y)}{|x-y|^{n}} f(y) d y,
$$

then

$$
\left\|T_{P}(f)\right\|_{\dot{F}_{p, q}^{s}} \leq C\|f\|_{\dot{F}_{p, q}^{s}}
$$

and

$$
\left\|T_{P}(f)\right\|_{F_{p, q}^{s}} \leq C\|f\|_{F_{p, q}^{s}},
$$

where $C_{p, q}$ is a constant which depends only on $p, q$ and degree of the real polynomial $P(x)$ but not its coefficients, and $\dot{F}_{p, q}^{s}$ denotes the homogenous TriebelLizorkin space and $F_{p, q}^{s}$ denotes inhomogeneous versions of Triebel-Lizorkin space; see [21]. 
We remark that in the case $\Omega \in L \log ^{+} L\left(S^{n-1}\right)$, the inequality (4.14) in Corollary 4.1 has been proved by Chen et al. [7] by another way under the condition $\nabla P(0) \neq 0$. Moreover, Corollary 4.1 is also new even if in the case $\Omega \in B_{r}^{0, k}\left(S^{n-1}\right)$ with $r>1$.

Acknowledgement. The authors would like to thank the referee for some valuable suggestions.

\section{References}

[1] J. Alvarez, R. Bagby R, D. Kurtz, and C. Pérez, Weighted estimates for commutators of linear operator, Studia Math. 104 (1993), no. 2, 195-209.

[2] H. Al-Qassem and Y. Pan, $L^{p}$ estimates for singular integrals with kernels belonging to certain block spaces, Rev. Mat. Iberoamericana. 18 (2002), no. 3, 701-730.

[3] A. Al-Salman and Y. Pan, Singular integrals with rough kernels in $L \log L\left(S^{n-1}\right)$, J. London Math. Soc. 66 (2002), no. 1, 153-174.

[4] K. Andersen and R. John, Weighted inequalities for vector-valued maximal functions and singular integrals, Studia Math. 69 (1980/81), no. 1, 19-31.

[5] A. P. Calderón and A. Zygmund, On singular integrals, Amer. J. Math. 78 (1956), 289-309.

[6] Y. Chen and Y. Ding, Rough singular integrals on Triebel-Lizorkin space and Besov space, J. Math. Anal. Appl. 347 (2008), no. 2, 493-501.

[7] J. Chen, H. Jia, and L. Jiang, Boundedness of rough oscillatory singular integral on Triebel-Lizorkin spaces, J. Math, Anal. Appl. 306 (2005), no. 2, 385-397.

[8] R. Coifman and Y. Meyer, Avdéla des opérate pseudo-différentiles, Astérisque. 57 (1978), 1-185.

[9] J. Duoandikoetxea, Weighted norm inequalities for homogeneous singular integrals, Trans. Amer. Math. Soc. 336 (1993), no. 2, 869-880.

[10] J. Duoandikoetxea and J. Rubio de Francia, Maximal and singular integral operators via Fourier transform estimate, Invent. Math. 84 (1986), no. 3, 541-561.

[11] C. Fefferman and E. Stein, Some maximal inequalities, Amer. J. Math. 93 (1971), 107115.

[12] J. García-Cuerva and J. Rubio de Francia, Weighted Norm Inequalities and Related Topics, Amsterdam-New York, North-Holland, 1985.

[13] S. Hofmann, Weighted norm inequalities and vector valued inequalities for certain rough operators, Indiana Univ. Math. J. 42 (1993), no. 1, 1-14.

[14] Y. Hou and L. Tang, $L^{p}\left(\mathbb{R}^{n}\right)$ boundedness for higher commutators of singular integrals with rough kernels belonging to certain block spaces, Math. Nachr. 282 (2009), no. 5, 713-725.

[15] G. Hu, $L^{p}\left(\mathbb{R}^{n}\right)$ boundedness for the commutators of homogeneous singular integral operators, Studia. Math. 154 (2003), no. 1, 13-27.

[16] G. Hu, S. Lu, and B. Ma, Commutators of convolution operators, Acta. Math. Sinica. 42 (1999), no. 2, 359-368

[17] S. Lu, M. Taibleson, and G. Weiss, Spaces Generated by Blocks, Beijing Normal University Press, 1989.

[18] S. Lu and $\mathrm{H}$. Wu, Oscillatory singular integrals and commutators with rough kernels, Ann. Sci. Math. Québec 27 (2003), no. 1, 47-66.

[19] S. Lu and Y. Zhang, Criterion on $L^{p}$-boundedness for a class of oscillatory singular integrals with rough kernels, Rev. Math. Iberoamericana 8 (1992), no. 2, 201-219.

[20] F. Ricci and E. Stein, Harmonic analysis on nilpotent groups and singular integrals I: Oscillatory integrals, J. Funct. Anal. 73 (1987), no. 1, 179-194.

[21] H. Triebel, Theory of Function Spaces, Basel, Birkhäuser, 1983. 
[22] D. Watson, Vector-valued inequalities, factorization, and extrapolation for a family of rough operators, J. Funct. Anal. 121 (1994), no. 2, 389-415.

LiN TANG

LMAM

School of Mathematical Sciences

Peking University

Beijing, 100871, P. R. China

E-mail address: tanglin@math.pku.edu.cn

HuOXIONG Wu

School of Mathematical Sciences

XiAmen University

Xiamen 361005, P. R. China

E-mail address: huoxwu@xmu.edu.cn 